\title{
polymer communications
}

Phase transformations of a liquid crystalline epoxy during curing*

\author{
Qinghuang Lin and Albert F. Yee† \\ Department of Materials Science and Engineering, The University of Michigan, Ann Arbor, \\ MI 48109-2136, USA
}

and Jimmy D. Earls, Robert E. Hefner Jr and Hung-Jue Sue

Texas Polymer Center, B-1470, Dow Chemical Co., Freeport, IX 77541, USA

(Received 9 December 1993; revised 20 January 1994)

\begin{abstract}
The phase transformations of a novel liquid crystalline epoxy, diglycidyl ether of 4,4'-dihydroxy- $\alpha$ methylstilbene (DGEDHMS), from isotropic phase to liquid crystalline phase during curing have been studied using a cross-polarized optical microscope equipped with a hot-stage. Time-temperaturetransformation (TTT) diagrams were constructed following the isothermal curing of the DGEDHMS in the isotropic phase with a difunctional amine, the aniline adduct of the DGEDHMS, and a tetrafunctional sulfonamido amine, sulfanilamide. Our results show that curing with the difunctional amine results in a nematic-like texture, while curing with the tetrafunctional sulfonamido amine leads to a smectic-like texture. Furthermore, curing with the difunctional amine gives rise to a faster development of the liquid crystalline phase, but with a less stable mesophase as compared to the tetrafunctional curing agent. These results are explained in terms of intermolecular interaction and the chain conformation anisotropy resulting from the curing reactions. The TTT diagram will be useful for providing guidelines for processing these new liquid crystalline epoxy systems.
\end{abstract}

(Keywords: phase transformation; liquid crystalline epoxy; curing)

\section{Introduction}

Liquid crystalline thermosets (LCTs) are a new family of polymeric materials that may offer unique properties for various applications, such as matrices for high performance composites, electronic packaging and nonlinear optics. In the LCTs, the molecular orientational order of polymers or oligomers is preserved through thermal or photocrosslinking of the functional groups of the liquid crystalline precursors ${ }^{1-15}$. As a result, the LCTs may offer many advantages over current liquid crystalline thermoplastics. These advantages include much better processability and enhanced transverse mechanical properties due to the covalent crosslinks.

In a previous study, we synthesized a novel thermotropic liquid crystalline epoxy, diglycidyl ether of 4,4'-dihydroxy$\alpha$-methylstilbene (DGEDHMS) (Figure l). Curing the DGEDHMS with a tetrafunctional sulfonamido amine resulted in thermosets with good mechanical properties as well as excellent thermal and chemical resistance ${ }^{1}$. Recently, Barclay et al. ${ }^{2}$ reported characterization of the DGEDHMS and its derivatives and preparation of oriented liquid crystalline networks from the liquid crystalline epoxies. They found that the DGEDHMS was a monotropic nematogen which exhibited a mesophase on cooling from 106 to $64^{\circ} \mathrm{C}$. To produce networks with 'locked-in' molecular order of the liquid crystalline state, they heated the mixtures of the DGEDHMS and curing agents to the isotropic phase, and then lowered the temperature to the liquid crystalline state to execute the

\footnotetext{
* The information in this paper is presented in good faith, but no warranty, expressed or implied, is given, nor is freedom from any patent to be inferred

† To whom correspondence should be addressed
}

curing reaction. The resulting network showed a smecticlike structure.

In the current study we have found that isothermal curing at temperatures above the liquid crystalline range for the DGEDHMS also leads to the development of liquid crystallinity. In this communication we report the isothermal curing of the DGEDHMS with two curing agents in the isotropic phase and the phase transformation from the isotropic phase to liquid crystalline phase during curing. The effects of the curing reaction on liquid crystallinity are discussed.

\section{Experimental}

Materials. The liquid crystalline epoxy, the DGEDHMS, was synthesized at Dow Chemical Co., Freeport, TX, USA ${ }^{1}$. This liquid crystalline epoxy was used without further purification. Two curing agents were used in this study: one was a tetrafunctional sulfonamido amine, sulfanilamide (SAA); the other was a difunctional amine, the aniline adduct of the DGEDHMS (AADGEDHMS). This curing agent was also synthesized at the Dow Chemical Co. ${ }^{16}$. Chemical structures of the curing agents are depicted in Figure 1. Samples for curing ,studies were prepared by meltmixing a stoichiometric amount of the liquid crystalline epoxy with the curing agent. The mixtures were then immediately cooled down to room temperature and kept in a refrigerator until further use.

Cptical microscope. The optical microscope used was an Olympus HB-2 polarizing microscope equipped with a Mettler hot-stage and a central processor. A small amount of a mixture of the liquid crystalline epoxy and 


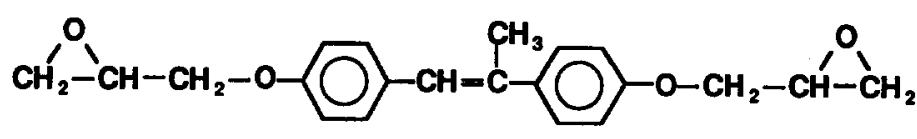

a<smiles>Nc1ccc(S(N)(=O)=O)cc1</smiles>

b

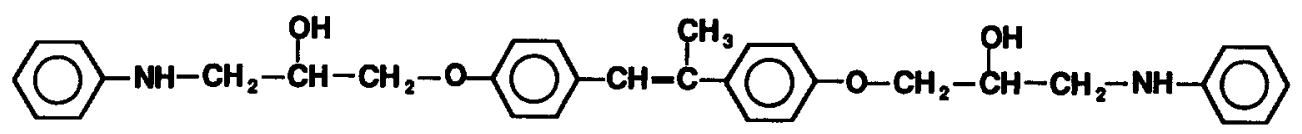

C

Figure 1 Chemical structures of the liquid crystalline epoxy and the curing agents. (a) DGEDHMS; (b) SAA; (c) aniline adduct of DGEDHMS

curing agent was put on a preheated glass slide. A cover glass was then placed on top of the sample and pressed to make a thin film of the sample. To reduce surface effects, the cover glass was removed before observation was started. The sample was then held isothermally for observing the development of liquid crystallinity during curing. The onset of massive birefringence was taken as the point of isotropic-liquid crystalline transformation.

Differential scanning calorimetry (d.s.c.). D.s.c. measurements were conducted on a Perkin-Elmer thermal analysis system (DSC-7) under a nitrogen atmosphere. Samples were sealed in aluminium pans and heated/cooled in the DSC-7 at a rate of $10^{\circ} \mathrm{C} \mathrm{min}^{-1}$. Sample weights ranged from 10 to $20 \mathrm{mg}$. The temperature was calibrated with an indium standard.

\section{Results and discussion}

The liquid crystalline epoxy, DGEDHMS, is a monotropic nematogen. Upon heating, it melts directly from a crystalline state into an isotropic state at $128^{\circ} \mathrm{C}$. Upon cooling from the isotropic state, it shows a nematic phase from 95 to $46^{\circ} \mathrm{C}$. In the liquid crystalline state, the epoxy shows a typical nematic schlieren texture (Figure 2). Mixtures of the DGEDHMS and the curing agents also exhibit similar monotropic mesophase behaviour with depressed transition temperatures.

When cooled from the isotropic phase $\left(130^{\circ} \mathrm{C}\right)$, the mixture of DGEDHMS and the difunctional curing agent enters into a nematic phase at $84^{\circ} \mathrm{C}$, and begins to crystallize at $74^{\circ} \mathrm{C}$. The reduced isotropic-nematic transition temperature is due to the dilution effect of the curing agent, and the increased crystallization temperature is due to a small amount of curing which occurs during melt-mixing and cooling. Above $84^{\circ} \mathrm{C}$, the DGEDHMS-curing agent mixture is an isotropic melt. Isothermal curing at temperatures between 84 and $160^{\circ} \mathrm{C}$ leads to the development of liquid crystallinity. The final products are opaque and show a nematic-like texture under a cross-polarized microscope (Figure 3). However, curing at temperatures above $160^{\circ} \mathrm{C}$, no mesophase formation is observed even after prolonged curing (up to $8 \mathrm{~h}$ ). The final products are transparent anci do not exhibit birefringence under the cross-polarized microscope. Curing of the DGEDHMS with the tetrafunctional curing

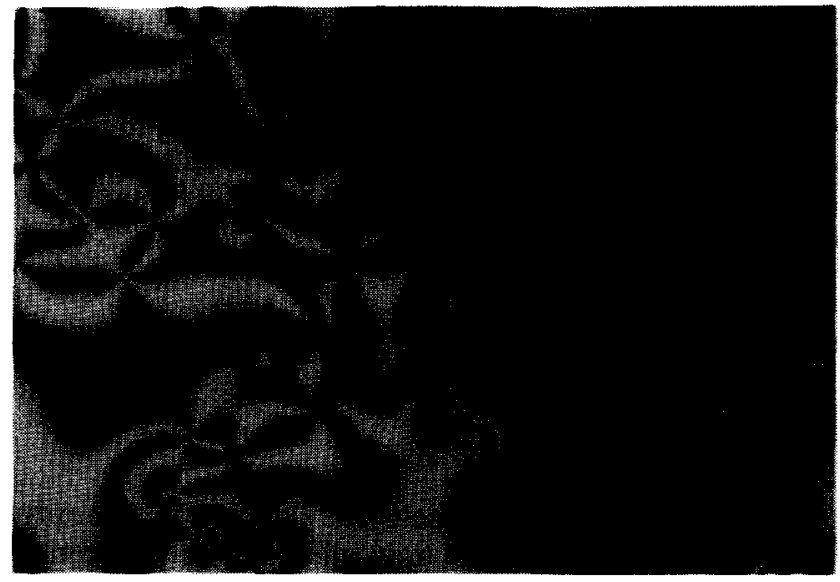

Figure 2 Schlieren texture of DGEDHMS at $91^{\circ} \mathrm{C}$ after cooling from isotropic phase $\left(140^{\circ} \mathrm{C}\right)$

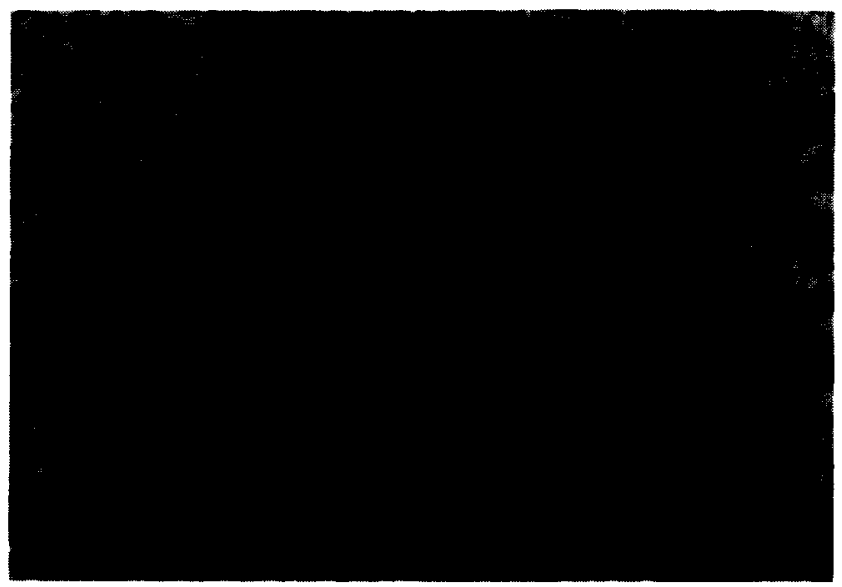

Figure 3 Polarized optical micrograph of cured DGEDHMS with the difunctional amine

agent, SAA, shows similar phenomena with the following two exceptions: the cured products exhibit a smectic-like texture (Figure 4), and the stability of the mesophase is raised by $20^{\circ} \mathrm{C}$ with the tetrafunctional curing agent.

In a theoretical analysis, Warner et al. ${ }^{17}$ concluded that the crosslinking of nematogenic polymers in different phases causes an opposite effect on the stability of a liquid 


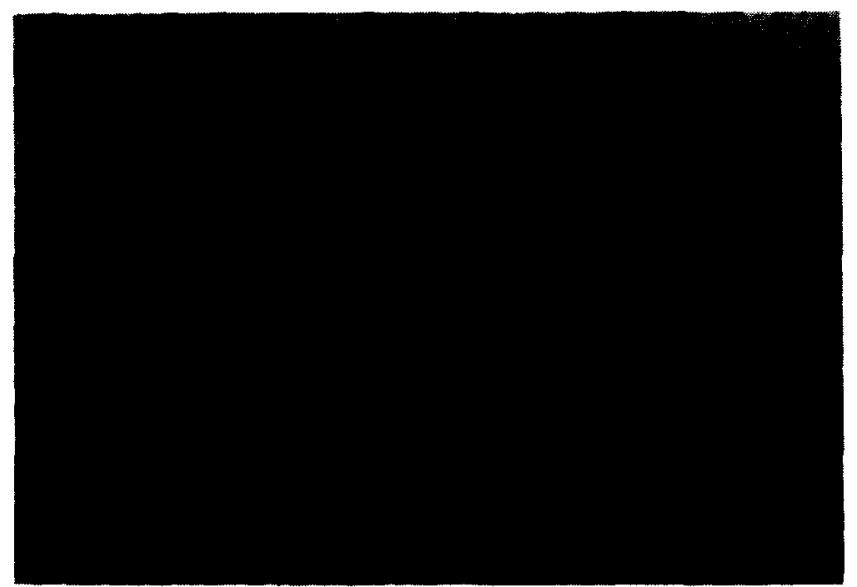

Figure 4 Polarized optical micrograph of cured DGEDHMS with the tetrafunctional sulfonamido amine

crystalline phase. Crosslinking in a nematic state raises the nematic-isotropic phase transition temperature compared with the uncrosslinked case, while crosslinking in an isotropic state lowers the transition temperature. In other words, crosslinking of a nematic polymer in the isotropic state will not lead to development of liquid crystallinity. However, Warner's analysis was restricted to crosslinking reactions without any chain extension. In the curing of the liquid crystalline epoxy, two types of structure changes can occur: one results from crosslinking and the other from chain extension. It is through chain extension that the liquid crystalline-isotropic phase transition temperature is raised when curing in the isotropic state.

Figures 5 and 6 show the time-temperature-transformation (TTT) diagrams from isotropic phase to liquid crystalline phase of the DGEDHMS-curing agent mixtures during isothermal curing. Both mixtures give an S-shaped TTT diagram, but the incipience of the liquid crystalline state for the system using the tetrafunctional curing agent is delayed by about 20 min compared to the system using the difunctional curing agent. This S-shaped TTT diagram is the result of the complicated interplay of the thermodynamics and curing kinetics of the reactive systems. The central issue here is how the curing reaction affects the liquid crystallinity of the reactive systems.

In general, thermotropic mesophases occur because of a delicate balance between the anisotropic dispersion interactions, the steric packing interactions and the thermal perturbation. The principal requirement for thermotropic mesomorphism is a pronounced molecular anisotropy ${ }^{18}$. The molecular anisotropy can be derived from conjugated aromatic molecular structures as well as non-spherical polymer chain conformations. The stability of the liquid crystal phase also depends heavily on the molecular anisotropy ${ }^{19}$. The phase transformation of the DGEDHMS will be interpreted on the basis of these understandings.

The development of liquid crystallinity from the isotropic phase is undoubtedly related to the reactive nature of the systems. The curing of the DGEDHMS with both curing agents gives rise to extended chain conformations. Therefore, liquid crystallinity ensues. However, from Figures 5 and 6 it is seen that the onset of the liquid crystalline state is faster with the difunctional curing agent than with the tetrafunctional curing agent.

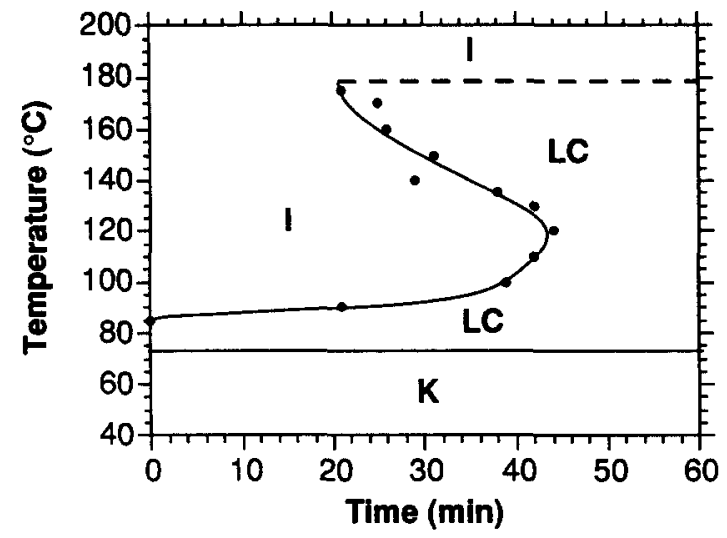

Figure 5 Time-temperature-phase transformation diagram of the isothermal curing of DGEDHMS with the tetrafunctional sulfonamido amine: I, isotropic; LC, liquid crystalline; $\mathrm{K}$, crystalline

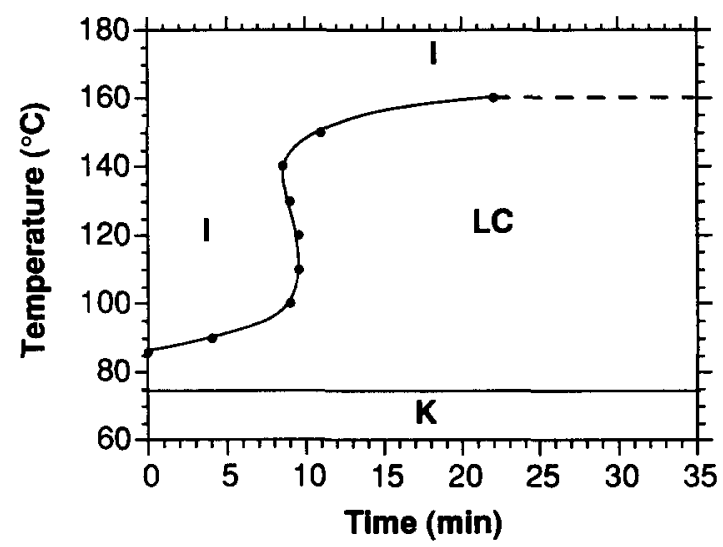

Figure 6 Time-temperature-phase transformation diagram of the isothermal curing of DGEDHMS with the difunctional amine (symbols as in Figure 5)

There are three possible reasons for the faster formation of mesophase with the difunctional curing agent: (a) the curing reaction proceeds faster with the difunctional curing agent; (b) curing with the difunctional curing agent results in more extended chain conformations which are inducive to the mesophase formation; (c) the combination of both effects.

We believe that chain conformations resulting from curing reaction play a more important role in determining the incubation time for the mesophase formation. On one hand, the curing reaction with the difunctional amine leads predominantly to chain extension. The possibility of a crosslinking reaction is very low because the reactivity of the backbone hydroxyl group, a product of reaction of the epoxide group with the amine group, is much lower than the amine group. On the other hand, the curing reaction with the tetrafunctional sulfonamido amine results in branched and crosslinked polymer chains, even though there exists a chain extension especially at lower curing temperatures and at the early stages of curing. As a consequence, the conformational anisotropy of the cured epoxy is higher for the system with the difunctional amine than for the system with the tetrafunctional sulfonamido amine. The higher conformational anisotropy, therefore, leads to earlier incipience of the liquid crystalline state at the same temperature. 
From Figures 5 and 6 it is also noted that the upper temperature limit for stable mesophase formation is $180^{\circ} \mathrm{C}$ for the system with the tetrafunctional curing agent, and that for the system with the difunctional curing agent the limit is around $160^{\circ} \mathrm{C}$. Curing with the tetrafunctional sulfonamido amine gives rise to a $20^{\circ} \mathrm{C}$ increase in mesophase stability. The enhanced mesophase stability with the tetrafunctional sulfonamido amine is due to the strong covalent bonding of polymer chains, which results from the crosslinking reaction. This strong covalent bonding makes it more difficult to break the liquid crystalline order. In other words, a stronger intermolecular interaction favours the stability of the mesophase.

From the above analysis it is obvious that, to foster the development of liquid crystallinity for the liquid crystalline epoxy, a curing agent must be selected such that sufficient chain extension takes place before crosslinking occurs. This will lead to the early induction of the liquid crystalline state and a higher mesophase stability. One approach is to use curing agents with reactive groups of different reactivities, so-called 'dual active' curing agents. The higher reactive group acts primarily as a chain extension agent while the lower reactive group functions as a crosslinking agent. The curing agent, SAA, is used here as an example of this kind of curing agent.

\section{Conclusion}

The phase transformations of the liquid crystalline epoxy, DGEDHMS, from isotropic phase to liquid crystalline phase during curing have been studied using a cross-polarized optical microscope equipped with a hot-stage. Time-temperature-transformation diagrams were constructed following isothermal curing of the DGEDHMS in the isotropic phase with a difunctional amine and a tetrafunctional sulfonamido amine. Our results show that curing with the difunctional amine results in a nematic-like texture, while curing with the tetrafunctional sulfonamido amine leads to a smectic-like texture. Furthermore, curing with the difunctional amine gives rise to a faster development of the liquid crystalline phase, but with a less stable mesophase as compared to the tetrafunctional curing agent. These results are explained in terms of intermolecular interaction and the chain conformation anisotropy resulting from the curing reactions. The TTT diagram will be useful for providing guidelines for processing these new liquid crystalline epoxy systems.

\section{Acknowledgements}

The authors are indebted to Professor D. C. Martin of the University of Michigan for helpful suggestions and comments, and to Drs J. L. Bertram, F. J. Knoll and L. J. Porter of The Dow Chemical Company for valuable assistance. The authors thank The Dow Chemical Company for permission to publish this work. The research at the University of Michigan is supported by The Dow Chemical Company.

\section{References}

1 Earls, J. D., Hefner, R. E. Jr and Puckett, P. M. Eur. Pat. Appl. EP 379,057, 1990

2 Barclay, G. G., Ober, C. K., Papathomas, K. I. and Wang, D. W. J. Polym. Sci., Polym. Chem. Edn 1992, 30, 1831, 1845

3 Broer, D. J., Lub, J. and Mol, G. N. Macromolecules 1993, 26 1244

4 Barclay, G. G., Ober, C. K., Papathomas, K. I. and Wang, D. W. Macromolecules 1992, 25, 2947

5 Anderson, A., Gedde, U. W. and Hult, A. Polymer 1992, 33, 4014

6 Litt, M. H., Whang, W.-T., Yen, K.-T. and Qian, X.-J. J. Polym. Sci., Polym. Chem. Edn 1993, 31, 183

7 Hikmet, R. A. M. Macromolecules 1992, 25, 5759

8 Hikmet, R. A. M., Lub, J. and Higgins, J. A. Polymer 1993, 34, 1736

9 Kishore, G. K. Macromolecules 1993, 26, 2995

10 Mallon, J. J. and Adams, P. M. J. Polym. Sci., Polym. Chem. Edn 1993, 31, 2249

11 Hoyt, A. E. and Benicewicz, B. C. J. Polym. Sci., Polym. Chem. Edn 1990, 28, 3403, 3417

12 Skrifvars, M. and Schmidt, H.-W. Am. Chem. Soc. Div. Polym. Chem. Polym. Prepr. 1993, 34 (2), 696

13 Douglas, E. P., Langlois, D. A. and Benicewicz, B. C. Am. Chem. Soc. Div. Polym. Chem. Polym. Prepr. 1993, 34 (2), 702

14 Mormann, W., Irle, Ch. and Zimmermann, J. Am. Chem. Soc. Div. Polym. Chem. Polym. Prepr. 1993, 34 (2), 704

15 Robinson, A. A., McNamee, S. C., Freidzon, Y. S. and Ober, C. K. Am. Chem. Soc. Div. Polym. Chem. Polym. Prepr. $1993,34(2), 743$

16 Hefner, R. E. Jr and Earls, J. D. Eur. Pat. Appl. EP 478,918, 1992

17 Warner, M., Gelling, K. P. and Vilgis, T. A. J. Chem. Phys. 1988 $88(6), 4008$

18 de Gennes, P. G. 'The Physics of Liquid Crystals', Oxford University Press, London, 1974

19 Flory, P. J. Proc. R. Soc. London, Ser. A 1956, 234, 73 